\title{
Formigas em Carcaças de Rattus norvegicus (Berkenhout) em uma Área de Cerrado no Sudeste do Brasil: Riqueza e Abundância
}

\author{
Alysson Rodrigo Fonseca ${ }^{\bowtie}$, Renata Bernardes Faria Campos \& Geiziane Ferreira Silva
}

Universidade do Estado de Minas Gerais UEMG/FUNEDI e-mail: arodrigofonseca@hotmail.com (Autor para correspondência ${ }^{\bowtie}$ ), rbfcampos@gmail.com, geizyferreira@yahoo.com.br.

\section{EntomoBrasilis 8 (1): 74-78 (2015)}

Resumo. Buscou-se conhecer as principais espécies de formigas associadas a carcaças de Rattus norvegicus (Berkenhout) ao longo do processo de decomposição, nas diferentes estações do ano em uma área de Cerrado. Em cada uma das quatro estações foi disposta em campo uma carcaça e ao redor desta foram instaladas cinco armadilhas do tipo pitfall. As formigas presentes na bandeja com a carcaça e nas armadilhas foram coletadas diariamente até o final da decomposição em cada uma das estações. Foram amostradas formigas pertencentes a vinte e três espécies, sendo $63,85 \%$ do total de indivíduos nas armadilhas e o restante coletado diretamente nas bandejas. Foram registradas a presença de espécies predadoras e nectarívoras. A escassez de recursos disponíveis no ambiente no período seco, possivelmente faz com que as carcaças sejam uma importante fonte de recursos, explicando a maior riqueza de espécies ativas nas proximidades da bandeja (coletadas nas armadilhas de queda). Esta maior atividade também se reflete em maior número de espécies e indivíduos nas carcaças. O oposto também parece ocorrer uma vez que na campanha de outono, final da estação chuvosa, notou-se a menor abundância de formigas nas carcaças. A presença de formigas nas carcaças ao longo de todas as fases do processo de decomposição e em todas as estações do ano evidenciou a importância da associação entre representantes deste táxon e carcaças em ambientes de Cerrado.

Palavras-Chave: Decomposição; Diversidade; Entomologia forense; Sucessão.

\section{Ants on Rattus norvegicus (Berkenhout) Carcass in a Savannah Area in Southeastern Brazil: Richness and Abundance}

Abstract. We studied the main species of ants associated with carcasses of Rattus norvegicus (Berkenhout) during the decomposition process, in different seasons in a savannah area. In each of the four seasons a carcass was disposed in the field and we installed around it five pitfall traps. Ants were collected from the tray containing the carcass and from the traps daily, until the end of decomposition in each season. Ants belonging to twentythree species, with $63.85 \%$ of the total individuals collected in the traps and the rest directly in the trays, were sampled. The presence of predatory and nectarivorous species were registered. The scarcity of available resources in the environment in the dry season possibly makes the carcasses important food sources, explaining the higher richness of active species near the trays (collected in the pitfall traps). This increased activity is also reflected in a higher number of species and individuals in the carcasses. The opposite also seems to occur once in the autumn, end of wet season, we observed lower abundance of ants in the carcasses. The presence of ants on carcasses throughout all stages of the decomposition process and in all seasons of the year highlighted the importance of the association between representatives of this taxon and carcasses in Cerrado environments.

Keywords: Decomposition; Diversity; Forensic entomology; Succession.

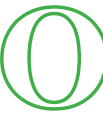
s estudos da entomofauna associada à decomposição de carcaças de vertebrados podem contribuir decisivamente para se conhecer a dinâmica de ciclagem de nutrientes nos ecossistemas, assim como a biodiversidade relacionada a estes processos (Oliveira-Costa 2008). Dentre os insetos, representantes das Ordens Diptera, Coleoptera, Hymenoptera, Lepidoptera, Blattodea, Hemiptera, Isoptera e Dermaptera, são apontados como os principais grupos que colonizam carcaças (Oliveira-Costa 2008). No Brasil, estudos nessa área têm sido realizados há mais de um século, sendo as moscas e os besouros apontados como os mais importantes (CARVAlHO et al. 2000; BARRos et al. 2008). Entretanto, grupos como as formigas (Hymenoptera: Formicidae) têm sido menos pesquisados, embora estudos abordem a importância destes insetos no processo de decomposição cadavérica no Brasil (CARVALHO et al. 2000; Moretti \& Ribeiro 2006; FonseCa et al. 2009; MoretTi et al 2008, 2013) e em outros países (EARLY \& GoFF 1986; VELÁsQUEZ 2008; BONACCI et al. 2011; CASTRO et al 2014).

Por serem muitas vezes generalistas, as formigas podem utilizar carcaças como refúgio, fonte de calor, umidade, fonte direta ou indireta de alimentação. Podem, portanto, utilizar diretamente a carcaça (tecidos, exsudados e couro) para a sua alimentação ou predar outros organismos que se alimentam diretamente da carcaça (EARly \& GofF 1986; Martinez et al. 1997; MoretTi \& Ribeiro 2006; Santos-Junior et al. 2014). Por isso, elas têm sido apontadas como importantes produtoras de ferimentos e predadoras de ovos e larvas de moscas em carcaças (Moura et al. 1997; Bonacci et al. 2011). Esses ferimentos têm sido analisados com cautela na área da entomologia forense, visto que pode levar os peritos a confundir lesões causadas pós-morte por formigas com lesões pré-morte ocasionadas por outros fatores, como escoriações causadas por objetos abrasivos ou pontiagudos (CAmpobasso et al. 2009).

A utilização de carcaças por determinadas espécies de formigas pode estar associada ao estágio de decomposição (Moura et al. 1997) e/ou ao ambiente em que se encontram (VELÁSQUEZ 2008). O efeito das formigas no processo de decomposição dependerá

Agências de Financiamento: Programa de Apoio à Pesquisa da Universidade do Estado de Minas Gerais (PAPq/UEMG), FAPEMIG e CNPq.

1 
T de sua identidade e abundância, bem como a área geográfica em questão (СAmpoBAsso et al. 2009). Nesse sentido, cada bioma tem sua fauna e condições locais próprias, o que exige o estudo das entomofaunas regionais e seus padrões de sucessão em carcaças, devendo as estimativas de riqueza, abundância e/ou diversidade serem aferidas de acordo com os padrões regionais e locais (BARros et al. 2008). Além disso, ao menos em ambientes de floresta secundária, sabe-se que o número de famílias de insetos depende do tamanho da carcaça e é maior na primavera (MoRETTI et al. 2013). Ainda assim, os dados disponíveis sobre ecologia e padrões de sucessão na fauna cadavérica são em sua maioria precários, sendo desconhecidos por nós, estudos que consideram a variação sazonal de formigas em cadáveres em ambientes de Cerrado.

Considerando a importância das formigas na comunidade sarcossaprófaga e a escassez de estudos sobre formicídeos associados a carcaças de vertebrados no Brasil, o presente trabalho teve como objetivo estudar a comunidade de formigas em carcaças de Rattus norvegicus (Berkenhout), em uma área de Cerrado. Buscou-se conhecer as principais espécies de formigas ao longo do processo sucessional nas diferentes estações do ano.

A pesquisa foi conduzida no Parque do Gafanhoto, uma área de preservação ambiental de 19,2 ha, localizada na periferia da cidade de Divinópolis ( $20^{\circ} 08^{\prime} 21^{\prime \prime S}, 44^{\circ} 53^{\prime} 17^{\prime \prime} \mathrm{O}, 718 \mathrm{~m}$ acima do nível do mar), centro-oeste de Minas Gerais. O clima é do tipo subtropical, com invernos secos e verões chuvosos. A temperatura média do inverno é de $16^{\circ} \mathrm{C}$ e do verão em torno dos $25^{\circ} \mathrm{C}$. A microrregião de Divinópolis apresenta um índice pluviométrico anual entre 1.100 e $1.700 \mathrm{~mm}$, sendo os meses entre dezembro e fevereiro os mais chuvosos. Os meses mais secos vão de abril a setembro (SEPLAN 2004).

A metodologia utilizada foi adaptada a partir daquela proposta por Mise et al. (2007), sendo as coletas realizadas em quatro etapas ao longo de um ano. Cada etapa ocorreu em uma estação, sendo que no ano de 2008 foram dispostas três carcaças (uma no mês de abril, correspondente ao outono, outra em julho correspondendo ao inverno, e a terceira em novembro, correspondendo à primavera) e uma quarta carcaça em março de 2009 (correspondendo ao verão).

Em cadaetapa, uma carcaçade $R$.norvegicuscompesoaproximado de $300 \mathrm{~g}$ teve sua decomposição acompanhada diariamente. Para isso, os roedores foram insensibilizados e posteriormente sacrificados mecanicamente, evitando extravasamento de fluidos. A carcaça foi exposta em uma área do Cerrado, inserida em uma gaiola ( $36 \times 30 \times 16 \mathrm{~cm})$ de metal e coberta por uma malha preta de $2,5 \mathrm{~cm}$ para permitir o acesso dos insetos e ao mesmo tempo impedir a ação de predadores de maior porte. A gaiola foi disposta dentro de uma bandeja plástica $(50 \times 40 \mathrm{~cm})$ e ao redor da mesma, foram colocadas cinco armadilhas do tipo pitfall (20 cm de diâmetro e $10 \mathrm{~cm}$ de profundidade), contendo solução de água, sal e detergente, enterradas ao nível do solo a uma distância de aproximadamente um metro da bandeja.

O conjunto "bandejas e armadilhas" foi vistoriado diariamente, entre as $13 \mathrm{e} 17 \mathrm{~h}$. Todos os espécimes capturados pelas armadilhas e no interior da bandeja foram coletados com auxílio de pinças entomológicas.

Para a discussão das fases de decomposição cadavérica, utilizouse o modelo de Bornemissza (1957), que divide o processo em cinco estágios: inicial (fresco), putrefação, putrefação escura, fermentação e seco, sendo a duração, em número de dias, registrada para cada fase. Ao longo de cada etapa, a temperatura na área do experimento foi medida diariamente, com o auxílio de um termômetro ambiente analógico, posicionado próximo à carcaça, para determinar as variações de temperatura ocorridas no local em um período de $24 \mathrm{~h}$, fornecendo as temperaturas máxima, mínima em cada dia de coleta.
O número de espécimes (abundância) coletados em cada vistoria foi registrado, bem como sua riqueza (número de espécies). Os espécimes coletados foram separados por subfamílias conforme a classificação de Bolton (2003) e posteriormente identificados até o menor nível taxonômico possível por comparação com a coleção de referência do Laboratório de Ecologia da UFV e depositados na coleção de referência da Universidade Estadual de Minas Gerais UEMG/FUNEDI.

Ao longo das quatro estações do ano foram coletadas 1.159 formigas, pertencentes a seis subfamílias, distribuídas em vinte e três espécies (Tabela 1). Armadilhas tipo pitfall coletaram 63,85\% $(\mathrm{n}=740)$ do total de indivíduos, sendo os $36,15 \%(\mathrm{n}=419)$ indivíduos restantes coletados diretamentenas bandejas. Somente duas espécies, Dolichoderus validus (Kempf) e Crematogaster sp, foram coletadas exclusivamente nas bandejas. Dez espécies foram coletadas exclusivamente das armadilhas de queda, enquanto outras onze espécies foram registradas pelos dois métodos. Desta forma, $56 \%$ das espécies coletadas estiveram em contato direto com a carcaça. Além das espécies coletadas exclusivamente nas bandejas é interessante notar que Camponotus sericeiventris (Guérin-Méneville) foi mais abundante na bandeja do que nas armadilhas de queda, evidenciando a influência da presença da carcaça sobre a atividade das formigas.

O período de decomposição das carcaças durou 19 dias no inverno, quando a temperatura média foi menor $\left(19,3^{\circ} \mathrm{C} \pm 0,2\right)$. Neste período foram coletadas seis espécies de formigas na bandeja e oito nas armadilhas de queda. No outono, primavera e verão o processo levou oito dias cada, sendo as temperaturas médias, respectivamente, $24,1^{\circ} \mathrm{C}\left( \pm 0,1^{\circ} \mathrm{C}\right), 23,6^{\circ} \mathrm{C}\left( \pm 0,3^{\circ} \mathrm{C}\right)$ e $25,9^{\circ} \mathrm{C}$ $\left( \pm 0,3^{\circ} \mathrm{C}\right)$. Três espécies foram coletadas na bandeja durante a decomposição no outono, seis espécies na primavera e nenhuma no verão, enquanto nas armadilhas de queda coletamos sete espécies no outono e nove em cada uma das outras duas estações. Foram coletadas 638 formigas no inverno, 186 nas coletas do verão, 176 no outono, e na estação da primavera 159 indivíduos foram coletados.

Considerando-se as quatro etapas de coletas reunidas, o número total de espécies coletadas em cada fase variou entre 20 no estágio de putrefação escuro e seis no estágio de fermentação, sendo onze espécies coletadas nos estágios de decomposição inicial e putrefação, e oito espécies coletadas no estágio seco. A variação na riqueza de formigas entre as fases não evidenciou um padrão de aumento ou redução ao longo da decomposição.

A presença de formigas ao longo de todas as fases do processo de decomposição em todas as estações do ano evidenciou a importância da associação destes organismos e carcaças em ambientes de Cerrado. No presente trabalho verificou-se grande abundância, diversidade e atividade destes organismos sobre as carcaças, corroborando com autores que afirmam que formigas têm importância forense (Wolf et al. 2004; Moretti \& Ribeiro 2006; Fonseca et al. 2009; BonacCi et al. 2011; MoretTi et al. 2013; SANTOS-Junior et al. 2014).

Diferente de Diptera e Coleoptera, que apresentam, respectivamente, picos de abundância no início e no final da decomposição (CARVAlHo et al. 2000), neste estudo as formigas foram registradas do início ao fim do processo. A ausência de um padrão consistente de alteração na abundância entre fases pode ser explicada pelo hábito generalista das formigas (MARTINEZ et al. 1997).

Não houve diferença em relação a riqueza de espécies e as fases de decomposição. A presença de formigas das mesmas espécies ao longo de todo o processo de decomposição já foi constatada por outros pesquisadores no Brasil, mas a abundância apresentou padrão distinto. Souza et al. (2008) registraram aumento das populações de Pheidole sp. e Camponotus rufipes (Fabricius) ao longo das fases de decomposição de carcaças de coelhos no 
Tabela 1. Abundância de formigas associadas a carcaças de R. novergicus no Parque do Gafanhoto, Divinópolis, MG em cada uma das estações do ano. O número de espécimes coletados por procura ativa na bandeja contendo as carcaças e nas armadilhas de queda são indicados, assim como as fases de decomposição em que os espécimes foram coletados (F1: inicial; F2: putrefação; F3: putrefação escuro; F4: fermentação; F5: seco).

\begin{tabular}{|c|c|c|c|c|c|}
\hline Subfamília & Espécie & Estação & Bandeja & Pitfall & Fase \\
\hline Dolichoderinae & Dolichoderus validus (Kempf) & Outono & 37 & - & $\mathrm{F} 2, \mathrm{~F} 3$ \\
\hline Ecitoninae & Labidus praedator (Smith) & Inverno & 2 & 291 & $\mathrm{~F} 1, \mathrm{~F} 2, \mathrm{~F} 3$ \\
\hline \multirow{3}{*}{ Ectatomminae } & Gnamptogenys striatula Mayr & Inverno & 4 & 10 & $\mathrm{~F} 1, \mathrm{~F} 2, \mathrm{~F} 3$ \\
\hline & & Primavera & - & 3 & $\mathrm{~F} 1, \mathrm{~F} 2$ \\
\hline & & Verão & - & 10 & $\mathrm{~F} 1, \mathrm{~F}_{4}, \mathrm{~F} 5$ \\
\hline \multirow{14}{*}{ Formicinae } & Camponotus ager (Smith) & Inverno & - & 1 & $\mathrm{~F}_{4}$ \\
\hline & & Primavera & 4 & 6 & $\mathrm{~F}_{1}, \mathrm{~F}_{2}, \mathrm{~F}_{3}, \mathrm{~F}_{5}$ \\
\hline & & Verão & - & 2 & $\mathrm{~F}_{4}$ \\
\hline & Camponotus atriceps (Smith) & Outono & - & 7 & F1 \\
\hline & & Inverno & - & 2 & $\mathrm{~F}_{3}$ \\
\hline & Camponotus blandus (Smith) & Outono & - & 2 & $\mathrm{~F}_{5}$ \\
\hline & Camponotus melanoticus Emery & Inverno & - & 3 & $\mathrm{~F}_{1}, \mathrm{~F}_{3}$ \\
\hline & Camponotus sericeiventris (Guérin-Méneville) & Primavera & 76 & 4 & $\mathrm{~F} 1, \mathrm{~F} 2, \mathrm{~F} 3, \mathrm{~F} 4, \mathrm{~F} 5$ \\
\hline & & Verão & 81 & - & $\mathrm{F}_{1}, \mathrm{~F} 2, \mathrm{~F}_{3}, \mathrm{~F}_{4}, \mathrm{~F}_{5}$ \\
\hline & Camponotus sp.1 & Inverno & 1 & 6 & F1, F3 \\
\hline & Camponotus sp.2 & Primavera & - & 6 & $\mathrm{~F}_{3}$ \\
\hline & Camponotus sp.3 & Inverno & - & 6 & F2 \\
\hline & Camponotus sp.4 & Verão & - & 5 & $\mathrm{~F}_{3}$ \\
\hline & Nylanderia sp & Outono & - & 4 & F3 \\
\hline \multirow{13}{*}{ Myrmicinae } & Atta sexdens (Linnaeus) & Outono & 7 & 20 & $\mathrm{~F}_{1}, \mathrm{~F} 2, \mathrm{~F}_{3}, \mathrm{~F}_{4}, \mathrm{~F}_{5}$ \\
\hline & & Inverno & 18 & 116 & $\mathrm{~F}_{1}, \mathrm{~F}_{2}, \mathrm{~F}_{3}, \mathrm{~F}_{4}, \mathrm{~F}_{5}$ \\
\hline & & Primavera & 7 & 22 & $\mathrm{~F}_{1}, \mathrm{~F}_{2}, \mathrm{~F}_{3}, \mathrm{~F}_{5}$ \\
\hline & & Verão & - & 61 & $\mathrm{~F} 1, \mathrm{~F} 2, \mathrm{~F} 3, \mathrm{~F} 4, \mathrm{~F} 5$ \\
\hline & Cephalotes clypeatus (Fabricius) & Inverno & 1 & 1 & $\mathrm{~F}_{3}$ \\
\hline & Crematogaster sp. & Inverno & 176 & - & $\mathrm{F}_{2}, \mathrm{~F}_{3}$ \\
\hline & Pheidole sp.1 & Primavera & 1 & 8 & $\mathrm{~F}_{1}, \mathrm{~F}_{3}, \mathrm{~F}_{5}$ \\
\hline & & Verão & - & 18 & $\mathrm{~F} 1, \mathrm{~F}_{4}, \mathrm{~F}_{5}$ \\
\hline & Pheidole sp.2 & Verão & - & 4 & F2 \\
\hline & Pheidole sp.3 & Primavera & - & 5 & $\mathrm{~F}_{2}, \mathrm{~F}_{3}$ \\
\hline & Pheidole sp.4 & Primavera & 1 & 13 & $\mathrm{~F}_{3}, \mathrm{~F}_{5}$ \\
\hline & & Verão & - & 4 & F5 \\
\hline & Solenopsis invicta Buren & Outono & - & 88 & $\mathrm{~F} 1, \mathrm{~F}_{3}, \mathrm{~F} 4$ \\
\hline \multirow{5}{*}{ Ponerinae } & Ondotomachus sp. & Outono & 2 & 3 & $F_{1}, F_{3}$ \\
\hline & Pachycondyla striata Smith & Outono & - & 6 & $\mathrm{~F}_{3}, \mathrm{~F}_{5}$ \\
\hline & & Primavera & 1 & 2 & $\mathrm{~F} 1, \mathrm{~F}_{3}, \mathrm{~F}_{5}$ \\
\hline & & Verão & - & 1 & $\mathrm{~F} 1$ \\
\hline & Total & & 419 & 740 & \\
\hline
\end{tabular}

Rio Grande do Sul. Diferentemente, encontramos picos de abundância nas fases iniciais de decomposição, no outono e especialmente no inverno.

O pico de abundância registrado no início do inverno, pode não ser um evento ligado à sazonalidade, e sim à presença estocástica de formigas de correição da espécie Labidus praedator (Smith), comuns na área estudada. Embora somente dois espécimes tenham sido coletados na bandeja, acreditamos que a passagem de maior número destas formigas pela carcaça evidenciouse principalmente pela presença massiva das mesmas nas armadilhas de queda dispostas ao seu redor e pelo fato de esta espécie movimentar-se em grupos compostos por grande número de indivíduos.

Neste sentido, o número de formigas eventuais e sua ação nas carcaças pode ser ainda maior do que documentamos aqui porque as vistorias ocorreram somente uma vez ao dia. Desta forma, o maior número de espécies encontrado nas armadilhas ao redor da gaiola pode evidenciar a atividade mais espécies nas proximidades da carcaça ao longo do dia. Esse resultado chama a atenção para a importância do conhecimento da fauna local em estudos forenses, uma vez que estas formigas removem quantidades consideráveis de biomassa e não permanecem num mesmo local por muito tempo, podendo confundir a interpretação de investigadores no que se refere, por exemplo, a ação de outros organismos necrófagos e ainda, a origem dos ferimentos detectadas nos cadáveres.

Por outro lado, salienta-se a influência da carcaça sobre a atividade das formigas, evidenciada pelo fato de que, ao analisarmos somente as formigas presentes nas bandejas notou-se diferença na composição de espécies em estações e fases distintas. Desta forma, no inverno, assim como na primavera, notou-se maior riqueza de espécies associadas à carcaça do que no verão e outono, resultado em acordo com a existência de duas estações (seca e chuvosa) ao longo do estudo. De modo distinto, outros estudos feitos em ambientes de Cerrado não encontraram variação na diversidade de formigas para as estações seca e chuvosa (MARQues \& Del-Claro 2006; ANdRAde et al. 2007). Os resultados deste estudo sugerem que, não só a menor temperatura, mas a provável redução das chuvas nesta época do ano faz com que carcaças sejam uma importante fonte de umidade para formigas no Cerrado. 
Estudos realizados na Península Ibérica na década de 90 apontaram a utilização de carcaças por formigas nectarívoras (MARTinez et al. 1997). Quase uma década depois foi registrado no sudeste brasileiro Cephalotes clypeatus (Fabricius) se utilizando de carcaças, em ambiente de mata (MoRETTI \& RibEIro 2006), fenômeno também registrado no presente trabalho para ambientes de Cerrado durante o inverno. A presença massiva do gênero tipicamente arbóreo, Crematogaster na carcaça e sua completa ausência nas armadilhas dispostas na circunvizinhança durante o inverno reforçam seu caráter "invasor de carcaças", já anunciado para Crematogaster scuttellaris (Olivier) (BonACCI et al. 2011). Além disso, estas formigas são parte de um dos principais gêneros que exploram recursos proteicos temporários em florestas semi-deciduais (SANTOS-Junior et al. 2014). O mesmo fenômeno foi encontrado no outono para D. validus, ainda não registradas em carcaças por outros autores.

Em Camponotus, seis das nove espécies foram coletadas somente nas armadilhas de queda. Entretanto, as seis espécies totalizam somente $23 \%$ dos espécimes amostrados. Esta discrepância deve-se à presença massiva de $C$. sericeiventris nas carcaças na primavera e no verão. $C$. sericeiventris dominou a carcaça nestas duas estações com raras ocorrências de formigas partilhando o recurso, enquanto no inverno Crematogaster dominou a carcaça reforçando o comportamento dominante destes grupos. Entretanto, Crematogaster ocupou a carcaça somente na Fase 2 (putrefação) e Fase 3 (putrefação escura), enquanto Camponotus parece ser menos específica, uma vez que esteve na bandeja em todos os estágios, nas duas estações em que foi coletada.

Por outro lado, Pheidole e Solenopsis, que são reconhecidas por seu habito oportunista e também já apontados como exploradores de fontes temporárias de proteínas (SANTOS-JUNIOR et al. 2014), não estiveram associadas diretamente às carcaças disponibilizadas neste estudo, exceto pela presença de dois dos 142 espécimes coletados.

Além da presença de formigas generalistas, nectarívoras e predadoras, notou-se a presença e utilização da carcaça por formigas cultivadoras de fungos. Formigas da tribo Attini diversificam o tipo de material coletado durante a estação seca (LEAL \& Oliveira 2000) e já foram observadas carregando corpos de outros insetos. Atta sexdens (Linnaeus) já foi coletada em armadilhas de queda nas proximidades de carcaças de suínos (CRUz \& VASCONCELOS 2006) e em carcaças de $R$. norvegicus (MoretTi et al. 2008).

Organismos herbívoros muitas vezes precisam de mais sódio do que conseguem obter a partir de fontes alimentares vegetais (KASPARi et al. 2008, Dudley et al. 2012, HernandÉZ 2012). Como resultado, muitos suplementam suas dietas com sódio adicional a partir de fontes não vegetais, como tecidos animais. De um modo geral, a escassez de recursos no período seco, possivelmente faz com que as carcaças sejam uma importante fonte de recursos, explicando a maior riqueza de espécies ativas (coletadas nas armadilhas de queda). Esta maior atividade também se reflete em maior número de espécies e indivíduos nas carcaças. O oposto também é verdade uma vez que no outono, ou seja, logo após a estação chuvosa cuja disponibilidade de recursos é maior, notouse a menor abundância de formigas nas carcaças.

O presente artigo confirma a presença de uma gama variada de espécies de formigas em carcaças de ratos e relata a utilização da carcaça por espécies que normalmente coletam matéria vegetal, como é o caso de $A$. sexdens, que foram observadas removendo partes da cauda do cadáver de $R$. norvegicus. A presença e ação destas formigas nas carcaças sugerem a necessidade de mais estudos visando elucidar o papel desses organismos na decomposição cadavérica. Os dados aqui apresentados permitem concluir que a relação das formigas com cadáveres no Cerrado pode estar sendo subestimada, uma vez que formigas são um grupo de insetos abundantes em praticamente todos os ecossistemas (HOLLDOBLER \& Wilson, 1990) e mais da metade das espécies coletadas em armadilhas de queda estiveram também presentes nas carcaças.

\section{AGRADECIMENTOS}

À Débora Silva Teixeira Borges e Marcio Cleib pelo auxílio nas coletas. Cinthia B. C. Milanez e Conrado A. Vieira pelo auxílio na preparação do manuscrito. Ao Programa de Apoio à Pesquisa da Universidade do Estado de Minas Gerais (PAPq/UEMG), FAPEMIG e CNPq pelo suporte financeiro. Aos revisores anônimos desse manuscrito, pela preciosa contribuição.

\section{REFERÊNCIAS}

Andrade, T., G.D.V. Marques \& K. Del-Claro, 2007. Diversity of ground dwelling ants in cerrado: an analysis of temporal variations and distinctive physiognomies of vegetation (Hymenoptera:Formicidae). Sociobiology, 50: 121-134.

Barros, R.M., C.A. De Mello-Patiu \& J.R. Pujol-Luz, 2008. Sarcophagidae (Insecta, Diptera) associated to the decay process of Sus scrofa Linnaeus (Suidae) carcasses in a Cerrado area of Distrito Federal, Brazil. Revista Brasileira de Entomologia, 52: 606-609.

Bolton, B., 2003. Synopsis and classification of Formicidae. Gainesville, The American Entomological Institute, 370p.

Bonacci, T., T.Z. Brandmayr, P. Brandmayr, V. Vercillo \& F. Porcelli, 2011. Successional patterns of the insect fauna on a pig carcass in southern Italy and the role of Crematogaster scutellaris (Hymenoptera, Formicidae) as a carrion invader. Entomological Science, 14: 125-132.

Bornemissza, G.F., 1957. An analysis of arthropod succession in carrion and the effect of its decomposition on the soil fauna. Australian Journal of Zoology, 5: 1-12.

Campobasso, C.P., D. Machetti, F. Introna \& M.F. Colonna, 2009. Postmortem artifacts made by ants and the effect of ant activitiy on decompositional rates. American Journal of Forensic Medicine and Pathology, 30: 84-87.

Carvalho, L.M.L., P.J. Thyssen, A.X. Linhares \& F.A.B. Palhares, 2000. A checklist of arthropods associated with pig carrion and human corpses in Southeastern Brazil. Memórias do Instituto Oswaldo Cruz, 95: 135-138.

Castro, C.P., M-D García, C. Palma \& M-D. Martínez-Ibánez, 2014. First report on sarcosaprophagous Formicidae from Portugal (Insecta: Hymenoptera). Annales de la Société entomologique de France (N.S.): International Journal of Entomology, 50: 51-58.

Cruz, T.M. \& S.D. Vasconcelos, 2006. Entomofauna de solo associada à decomposição de carcaça de suíno em um fragmento de mata atlântica de Pernambuco, Brasil. Biociências, 14: 193-201.

Dudley, R., M. Kaspari \& S.P. Yanoviak, 2012. Lust for salt in the western Amazon. Biotropica, 44, p. 6-9.

Early, M. \& M.L. Goff, 1986. Arthropod succession patterns in exposed carrion on the island of Oahu, Hawaiian Islands, USA. Journal of Medical Entomology, 23: 520-531.

Fonseca, A.R., G.F. Silva, M.C. Pereira, N.R. Alves \& D.S.T. Borges, 2009. Formigas (Hymenoptera: Formicidae) associadas à decomposição de carcaça de Rattusnovergicus (Berkenhout, 1769). III Congresso Latino Americano de Ecologia. Disponível em: <http://www.seb-ecologia.org. $\mathrm{br} / 2009 /$ resumos clae/85.pdf $>$. [Acesso em: 08.12.2014].

Hölldobler, B.\& E. O. Wilson, 1990. The Ants. Harvard Belknap, Cambridge. 732p.

Kaspari, M., S.P.Yanoviak\& R. Dudley, 2008. On thebiogeography of salt limitation: a study of ant communities. Proceedings of the National Academy of Sciences, 105: 17848-17851.

Leal, I.R. \& P.S. Oliveira, 2000. Foraging ecology of attine ants in a Neotropical savanna: seasonal use of fungal substrate in the cerrado vegetation of Brazil. Insectes Sociaux, 47: 376-382.

Marques, G.D.V. \& K. Del-Claro, 2006. The ant fauna in a Cerrado area: the influence of vegetation structure and seasonality 
(Hymenoptera: Formicidae). Sociobiology, 47: 235-252.

Martínez, M.D., Arnaldos, M.I. \& M.D. García, 1997. Datos sobre la fauna de hormigas asociada a cadáveres (Hymenoptera: Formicidae). Boletín de la Asociación Española de Entomología, 21: 281-283.

Mise, K.M., L.M. Almeida \& M.O. Moura, 2007. Levantamento da fauna de Coleoptera que habita a carcaça de Sus scrofa L., em Curitiba, Paraná. Revista Brasileira de Entomologia, 51: 358-368.

Moretti, T.C. \& O.B. Ribeiro, 2006. Cephalotes clypeatus Fabricius (Hymenoptera: Fomicidae): hábitos de nidificação e ocorrência em carcaça animal. Neotropical Entomology 35: $412-415$.

Moretti, T.C., D.R. Solis \& W.A.C. Godoy, 2013. Ants (Hymenoptera: Formicidae) Collected with Carrion-Baited Traps in Southeast Brazil. The Open Forensic Science Journal, 6: $1-5$.

Moretti, T.C., O.B. Ribeiro, P.J. Thyssen \& D.R. Solis, 2008. Insects on decomposing carcasses of small rodents in a secondary forest in Southeastern Brazil. European Journal of Entomology, 105: 691-696.

Moura, M.O.; C. J. B. Carvalho \& E.L.A. Monteiro-Filho, 1997. A preliminary analysis of insects of medical-legal importance in Curitiba, state of Paraná. Memórias do Instituto Oswaldo Cruz, 92: 269-274.

Oliveira-Costa, J., 2008. Entomologia Forense: quando os insetos são vestígios. Campinas, Millennium, 420 p.
Santos-Junior, L.C., J.M. Saraiva, R. Silvestre \& W.F. AntonialliJunior, 2014. Evaluation of Insects that Exploit Temporary Protein Resources Emphasizing the Action of Ants (Hymenoptera, Formicidae) in a Neotropical Semi-deciduous Forest. Sociobiology, 6: 43-51.

Seplan, 2004. Secretaria Municipal de Planejamento: Prefeitura Municipal de Divinópolis. Anuário estatístico de Divinópolis. $89 \mathrm{p}$.

Souza, A.S.B., F.D. Kirst \& R.F. Krüger, 2008. Insects of forensic importance from Rio Grande do Sul state in Southern Brazil. Revista Brasileira de Entomologia, 52: 641-646.

Velásquez, Y.A., 2008. Checklist of arthropods associated with rat carrion in a mountane locality of northern Venezuela. Forensic Science International, 174: 67-69.

Wolff, M., A. Builes, G. Zapata, G. Morales \& M. Benecke, 2004. Detection of Parathion (o,o-diethyl o-(4nitrophenyl) phosphorothioate) by HPLC in Insects of Forensic Importance in Medellín, Colombia. Anil Aggrawal's Internet Journal of Forensic Medicine and Toxicology, 5: 6-11.

\section{Recebido em: 28/o7/2014}

Aceito em: 20/01/2015

\section{Como citar este artigo:}

Fonseca. A.R., R.B.F. Campos \& G.F. Silva, 2015. Formigas em Carcaças de Rattus norvegicus (Berkenhout) em uma Área de Cerrado no Sudeste do Brasil: Riqueza e Abundância. EntomoBrasilis, 8 (1): 74-78.

Acessível em: doi:10.12741/ebrasilis.v8i1.460
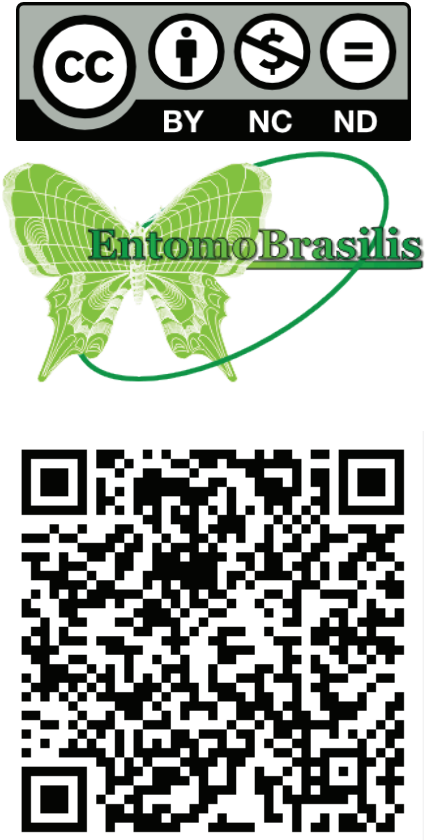